\title{
Fatherhood Following Treatment for Testicular Cancer: A Systematic Review and Meta-Analyses
}

\author{
Brigitte Gerstl, BSocSci, MPh, ${ }^{1,2}$ Michael J. Bertoldo, BScAgr (Hons), PhD, ${ }^{3,4}$ \\ Elizabeth Sullivan, MD, MPH, MMed, MBBS, FAFPHM, ${ }^{5}$ Xanthie Volckmar, BMSc, ${ }^{1}$ Aidan Kerr, BMSc, \\ Handan Wand, MS, MA, PhD, ${ }^{1}$ Angela Ives, Dip App Sc, MSc, PhD, ${ }^{6}$ \\ Olayan Albalawi, BMath, MMathSc, MSTAT ${ }^{1,7}$ and Antoinette Anazodo, MBBS, PhD $^{2,3,8}$
}

\begin{abstract}
Purpose: Testicular cancer (TC) is considered the most commonly diagnosed malignancy in males between 15 and 34 years of age. The objective of this study is to systematically review and meta-analyze studies on fatherhood following treatment for TC.

Methods: We reviewed studies reporting on fatherhood following TC from Medline and Embase search engines by developing search strategies. Only studies including patients with TC and at least one reproductive variable were considered as part of the analysis. Estimate of heterogeneity was calculated using the $I^{2}$ statistic. Metaanalyses employing a fixed effects model were also applied as an additional measure of sensitivity.

Results: A total of 27 studies were included which reported on fatherhood after treatment for TC. A meta-analysis of included studies with subgroup analysis was conducted. Subgroup analysis, for the combined studies, indicated an overall pooled pregnancy rate of $22 \%$ (95\% confidence intervals [CI]: $0.21-0.23 ; I^{2}=98.1 \%$ ) for couples who conceived after TC. Of those couples that became pregnant, $11 \%$ (95\% CI: $0.07-0.16 ; I^{2}=8.5 \%$ ) experienced a miscarriage. Fatherhood was experienced by $37 \%$ (95\% CI: $\left.0.35-0.39 ; I^{2}=98.1 \%\right)$ of males following treatment for TC.

Conclusions: Male cancer patients should be offered discussions, information, and counseling regarding the impact that TC treatment can have on fertility. Furthermore, sperm banking must be recommended to all patients before starting treatment.
\end{abstract}

Keywords: testicular cancer, fatherhood, oncofertility, reproductive outcomes, pregnancy, birth

\section{Background}

$\mathbf{T}$ ESTICUlar CANCER (TC) is considered the most commonly diagnosed malignancy in males between 15 and 34 years of age; however, this malignancy only accounts for $1 \%$ of all newly diagnosed male cancers. ${ }^{1}$ Patients with this diagnosis have a $95 \%$ five-year cancer-specific survival rate. ${ }^{2}$

More than $95 \%$ of testicular tumors are diagnosed as testicular germ cell tumors (TGCTs). ${ }^{3}$ Germ cell tumors (GCT) comprise of $\sim 98 \%$ of TCs. ${ }^{4}$ Other GCTs include stromal tumors $(2 \%)$, such as Leydig cell $(\sim 0.2 \%)$ and Sertoli cell tumors $(\sim 0.1)$. Approximately $55 \%$ of TGCTs are considered classic seminomas, $44 \%$ are nonseminomas (embryonal carcinomas, teratomas, yolk sac tumors, choriocarcinomas), and $1 \%$ are spermatocytic seminomas. ${ }^{4}$
The incidence of TC varies by ethnicity, with the highest rates reported in developed countries such as Norway (11.8/ $100,000)$ and the lowest in developing countries such as in India $(0.5 / 100,000)$ and Thailand $(0.4 / 100,000) .{ }^{5}$ There is an increased incidence of TGCTs diagnosed in young adult males, with most (84\%) diagnosed between the ages of 15 and 44 years. Less than $20 \%$, occur in males $\geq 45$ years of age. The incidence of nonseminoma peaks when a male is $\sim 25$ years of age, while the incidence of seminoma peaks in older males around the age 35 years. $^{6}$

Treatment outcomes can have variable effects on spermatogenesis, which is highly correlative to the quality of a patient's sperm before the start of gonadotoxic treatment as well as numerous other factors, including age at diagnosis, tumor type, drug characteristics (including novel drugs),

\footnotetext{
${ }^{1}$ Department of Biostatistics, The Kirby Institute, University of New South Wales, Sydney, Australia.

${ }^{2}$ Kids Cancer Center, Sydney Children's Hospital, Sydney, Australia.

${ }^{3}$ School of Women's and Children's Health, University of New South Wales, Sydney, Australia.

${ }_{5}^{4}$ School of Medical Sciences, University of New South Wales, Sydney, Australia.

${ }^{5}$ Faculty Health and Medicine, The University of Newcastle, Newcastle, Australia.

${ }^{6}$ Cancer and Palliative Care Research and Evaluation Unit, University of Western Australia, Crawley, Australia.

${ }^{7}$ Department of Statistics, Science College, Tabuk University, Tabuk, Saudi Arabia.

${ }^{8}$ Nelune Comprehensive Cancer Center, Prince of Wales Hospital, Sydney, Australia.
} 
treatment regimen, site of administration of treatment, and length of treatment. ${ }^{7}$ Radiotherapy and chemotherapy can either temporarily or permanently impair spermatogene$\mathrm{sis}^{8-11}$ and retroperitoneal lymph node dissection (RPLND) may be followed by dry ejaculation, in which surgery to sympathetic nerves and ganglia can cause loss of seminal emission into the posterior urethra or true retrograde ejaculation. ${ }^{12,13}$ The introduction of unilateral and/or nerve-sparing RPLND $^{14}$ and surveillance as primary therapy options after orchiectomy ${ }^{15}$ are considered appropriate treatment strategies to assist with preserving a patient's fertility. Other possible strategies for preserving fertility include limiting the radiation fields to the para-aortic area, ${ }^{16}$ lower doses of both radiation therapy ${ }^{17}$ and chemotherapy. ${ }^{18}$

Studies report that approximately a quarter of male cancer patients will experience azoospermia following treatment. ${ }^{19}$ Three quarters of patient's will present with a decrease in baseline spermatogenesis, ${ }^{11,20}$ mainly due to existing defects in spermatogenesis, a medical history of cryptorchidism, antisperm antibodies, as well as possible contralateral or intraepithelial germ cell neoplasia and generalized stress associated with illness. ${ }^{19,21}$ Studies conducted by Stephenson et al. ${ }^{22}$ and Matos et al. ${ }^{23}$ reported a drop in fertility rates, by half $(49 \%)$, following treatment with gonadotoxic therapy, with baseline fertility rates at $82 \%$ before the start of treatment.

Lampe et al. ${ }^{8}$ reported follow-up sperm counts of TC patients before and then 1 year post-treatment, revealing normal sperm count pre-treatment, with $16 \%$ presenting with oligospermic (<20 million sperms/mL) and $20 \%$ presenting azoospermic post-chemotherapy. Comparatively, it was also reported that in TC patients who presented with oligospermia before the start of treatment, only $24 \%$ had reached a normal sperm count 1 year post-treatment. ${ }^{8}$ Additionally, cryptorchidism, where one or both of the testes fail to descend from the abdomen into the scrotum, has been reported to be correlated with both TC and infertility. ${ }^{24,25}$

Since TC is most commonly diagnosed in males of reproductive age, at the peak of their reproductive potential, undergoing treatment with surgery, chemotherapy, radiotherapy, or a combination of therapies can have irreversible effects on becoming a father. ${ }^{26}$ Many studies report that male patients experience a number of clinical and psychosocial concerns in relation to their sexual and psychosexual dysfunction and subfertility or infertility following treatment. ${ }^{27,28}$ Surgery and radiation to the pelvis or lumbar spine has reportedly been linked to sexual dysfunction, as a result of hormonal deficiency and emotional distress. ${ }^{29}$ Kenney et al. ${ }^{29}$ reported that $20 \%$ of young adult male cancer patients experienced erectile dysfunction subsequent to treatment, and these findings were supported by several other studies revealing that more than half of all survivors presented with ejaculatory dysfunction following treatment. ${ }^{3,30}$ These consequential reproductive outcomes, in relation to fatherhood, can have a detrimental effect on a patient's physical and psychological wellbeing, maintaining relationships, and quality of life. ${ }^{31}$ Hence, discussion and counseling around sexuality, reproductive function, and fertility preservation are important before starting gonadotoxic treatment. ${ }^{32}$

Currently, the most common and efficient option for fertility preservation in males is sperm banking through masturbation, and its subsequent use in assisted reproductive technologies (ART), either with the use of in vitro fertilization or intracytoplasmic sperm injection, for those desiring a biological family. 7,33-37

Semen quality may be reduced before a patient undergoes treatment with an orchiectomy for TC and sperm count and concentration can further decrease after this procedure. $^{8,13,38-40}$ The European Society for Medical Oncology (ESMO) recommends patients who receive chemotherapy or radiotherapy following an orchiectomy should be encouraged to undergo sperm banking, as their fertility may be further decreased compared with those patients who undergo treatment with an orchiectomy alone. ${ }^{8,13,38-42}$ However, fatherhood may still be possible following treatment through natural conception or in vitro fertilization (IVF). ${ }^{42}$

The aim of this systematic review and meta-analysis was to investigate and report on fatherhood following treatment for a testicular malignancy in male patients.

\section{Methods}

\section{Search strategies}

This systematic review of the literature followed the Preferred Reporting Items for Systematic Review and MetaAnalyses (PRISMA) Statement (Fig. 1) and PROSPERO (CRD42019136085). Highly sensitive search strategies were designed for the databases MEDLINE (OVID) 1980-July 2019 and EMBASE 1980-July 2019. Searches were conducted using terms such as "testicular neoplasm," "testicular cancer," "testicular germ cell cancer," "seminoma," "nonseminoma," "teratoma," embryonal cancers," "choriocancers," "yolk sac tumors," "reproduction," “fertility,", "'fertilization," " "pregnancy," and "birth." Included articles were also checked for additional references. Both searches were limited to "English language" with "male", patients. Four authors (B.G., M.B., X.V., and A.K.) independently screened all citations and abstracts identified by the string search strategy to identify eligible studies. Reference lists, of articles that were screened, were checked to determine whether all pertinent literature was included.

\section{Criteria for inclusion of studies}

We first identified articles by examining titles, then abstracts to determine relevance, and then we retrieved the full text of the relevant abstracts for further assessment and review. Accordingly, we identified retrospective studies, population-based studies, cohort studies, randomizedcontrolled trials, and questionnaire studies (where data were presented on fatherhood that is the number of conceptions and/or subsequent birth outcomes). Among the criteria used in inclusion of studies were; (1) males diagnosed with a TC; (2) studies with adequate information on the method of ascertainment of fatherhood.; (3) studies where information was extracted from peer-reviewed journal articles; (4) medical record reviews or pathological reviews retrieved from participating sites described in the included articles; (5) questionnaires that presented data on reproductive outcomes for couples following $\mathrm{TC}^{43}$; (6) studies published between January 1980 and July 2019; (7) studies that had a sample size greater than or equal to 10 participants; and (8) full-text studies that were published in English. 
FIG. 1. PRISMA flow diagram of the study selection process. PRISMA, Preferred Reporting Items for Systematic Review and MetaAnalyses.

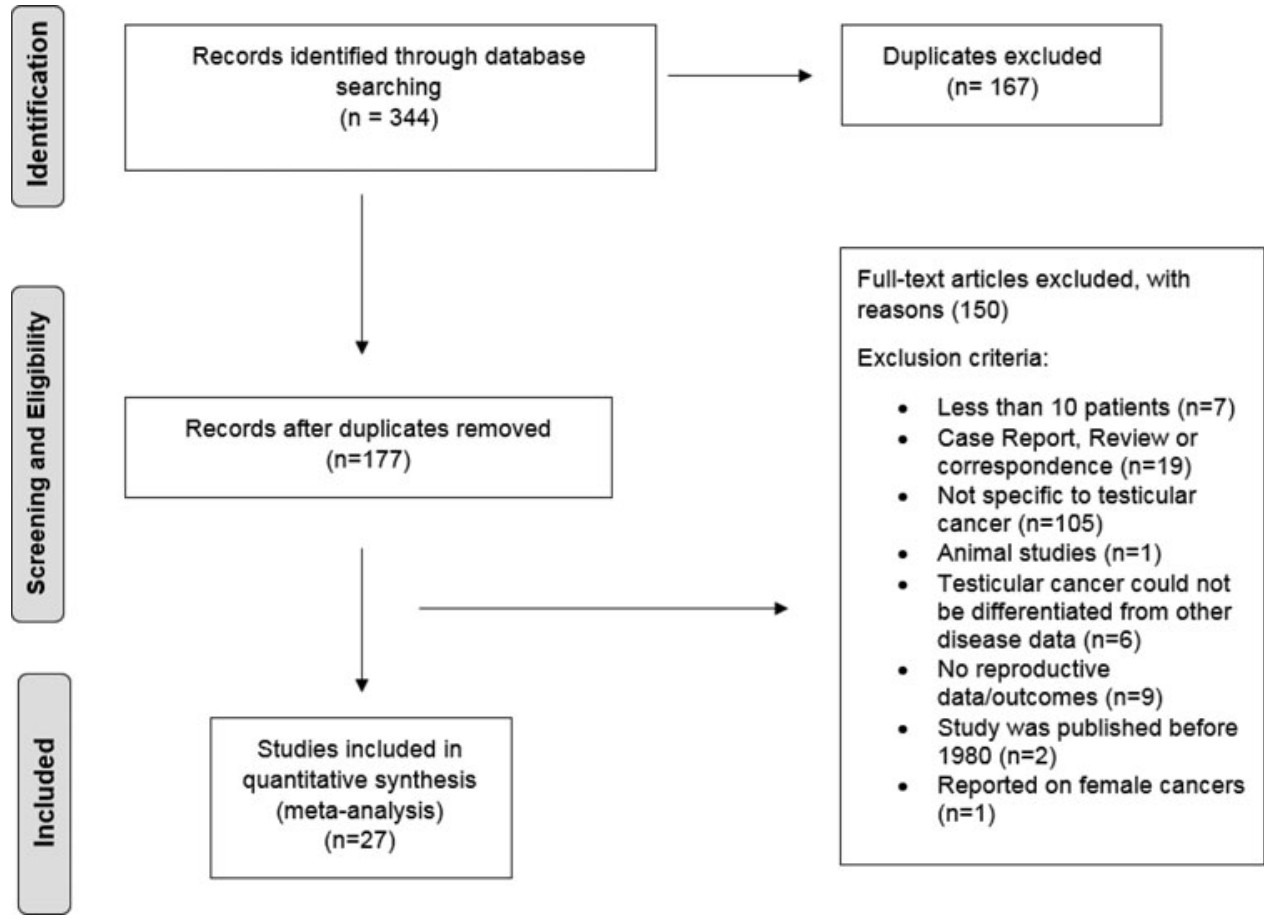

Studies were excluded: (1) if the study was not published in English and where we were unable to access a copy of the article translated in the English language; (2) qualitative articles that did not include reproductive data for males to be included in the analysis; (3) case studies, as most studies lacked robust quality reproductive data to include in the analysis and to reduce the potential for identifying a patient in this study; and (4) secondary works (e.g., review articles, commentaries, editorials, or dissertations/theses, or conference abstracts that had not yet been published).

\section{Data extraction}

The data collection tool was designed by two individuals (A.A., B.G.). Titles, abstracts, and keywords were independently reviewed by four individuals (B.G., M.B., X.V., and A.K.) and the full text for each included study was independently reviewed by four individuals (B.G., M.B., X.V., and A.K.). The full text was retrieved of any reference that gave an indication that it might contain data on at least one reproductive variable in relation to $\mathrm{TC}$.

\section{Data analysis}

Descriptive statistics were used to analyze the frequencies, medians, and mean of demographic data (age, study design, tumor type, and stage). Statistical analyses were performed using STATA release 14.2 (Stata Statistical Software; Stata Corporation, College Station, TX).

Meta-analyses. Meta-analyses were conducted using a random effects model to allow for variation in true associations across studies. ${ }^{44}$ Estimate of heterogeneity was calculated using the $I^{2}$ statistic, which is the percentage of total variation in risk estimates attributable to genuine variation rather than sampling error. ${ }^{45}$ Meta-analyses employing a fixed effects model were also applied as an additional mea- sure of sensitivity. ${ }^{46}$ Generally, heterogeneity is classified into the following three categories: heterogeneity less than $25 \%$ (low level of heterogeneity), between $25 \%$ and $75 \%$ (average level of heterogeneity), and more than $75 \%$ (high level of heterogeneity). Forest plots were used to present the combined estimate with $95 \%$ confidence intervals (CI). For studies which did not present a standard error (SE), we calculated SE using the formula; $\mathrm{SE}=\sqrt{\frac{\hat{p}(1-\hat{p})}{n}}$ in Microsoft excel.

\section{Results}

\section{Characteristics of included studies}

Initially, 344 publications were searched from databases and other resources. After screening potentially eligible studies, 27 studies ${ }^{13,30,32,33,35,38,39,41,43,47-64}$ met the inclusion criteria and full texts were subsequently retrieved. The studies included: three retrospective review studies $(11.11 \%)^{38,54,58}$; one prospective study $\left.(3.7 \%)\right)^{33}$ seven cohort studies $(25.93 \%),{ }^{13,49,56,57,60,62,64}$ four questionnaire studies $(14.81 \%),{ }^{47,48,50,59}$ three medical record review studies $(11.11 \%),{ }^{32,35,51}$ seven medical review and questionnaire studies $(25.93 \%),{ }^{30,39,52,53,55,61,63}$ one cross-sectional study $(3.7 \%),{ }^{41}$ and one registry study $(3.7 \%) .{ }^{43}$ Most studies were conducted in Europe $(n=15,55.56 \%),{ }^{13,30,32,38,39,41,43,48,50,56-60,62}$ followed by The Americas $(n=8,29.63 \%), 33,49,51-55,63$ Australasia $(n=3,11.11 \%),{ }^{47,61,64}$ and the Middle East $(n=1,3.70 \%) .^{35}$ Table 1 highlights study characteristic, including TC treatment details, for each of the 27 included studies. $13,30,32,33,35,38,39,41,43,47-64$

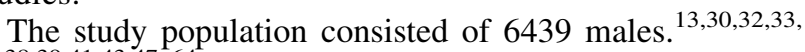
35,38,39,41,43,47-64 diagnosed with TC (seminomas $=2156$ $[33.48 \%]^{13,30,32,38,39,41,47-49,53,57-62} ; \quad$ nonseminoma $=3861$ $[59.96 \%]^{13,30,32,38,41,43,47-50,54,55,57-63}$; and unspecified $=$ $422[6.56 \%])$. 


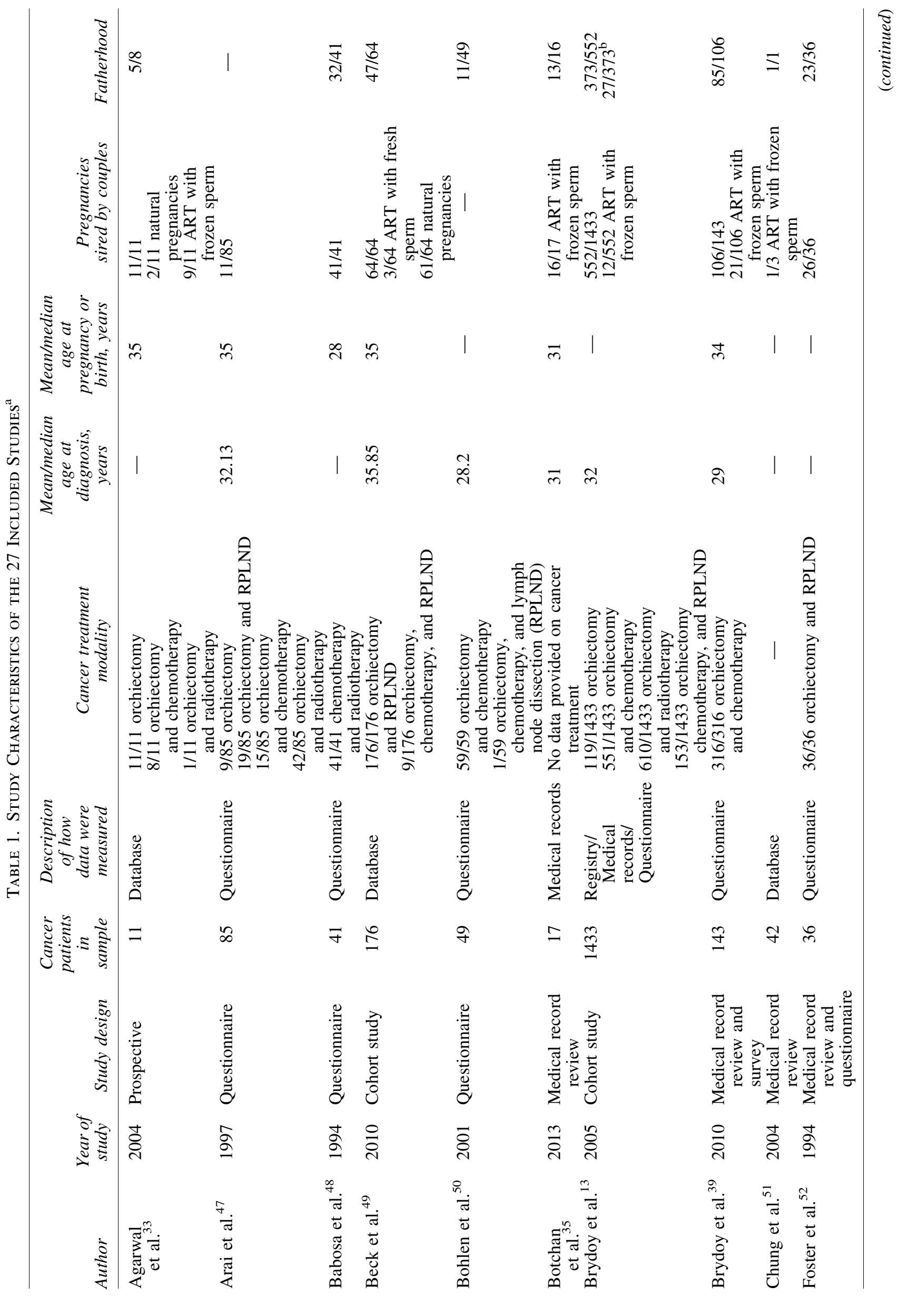




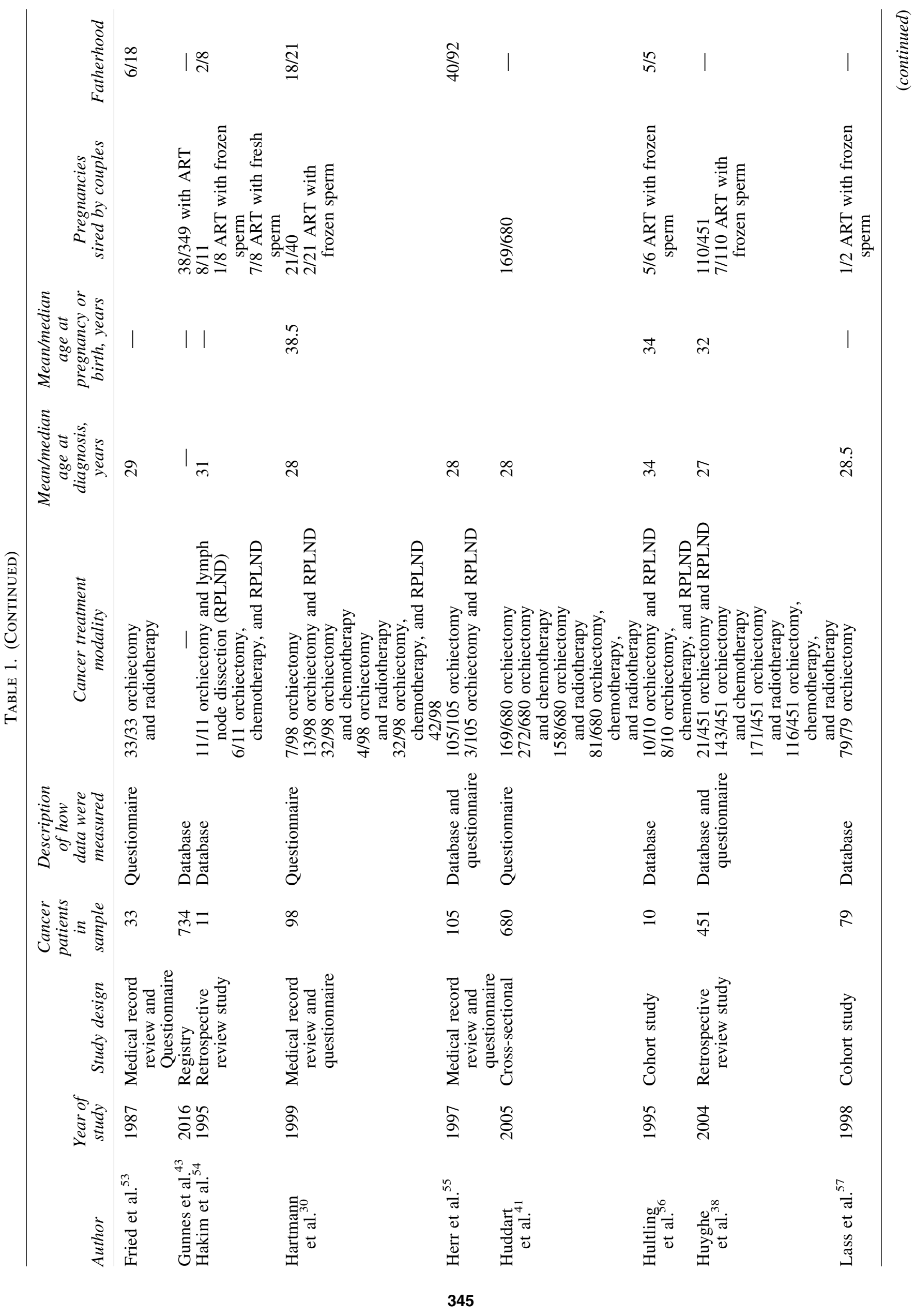




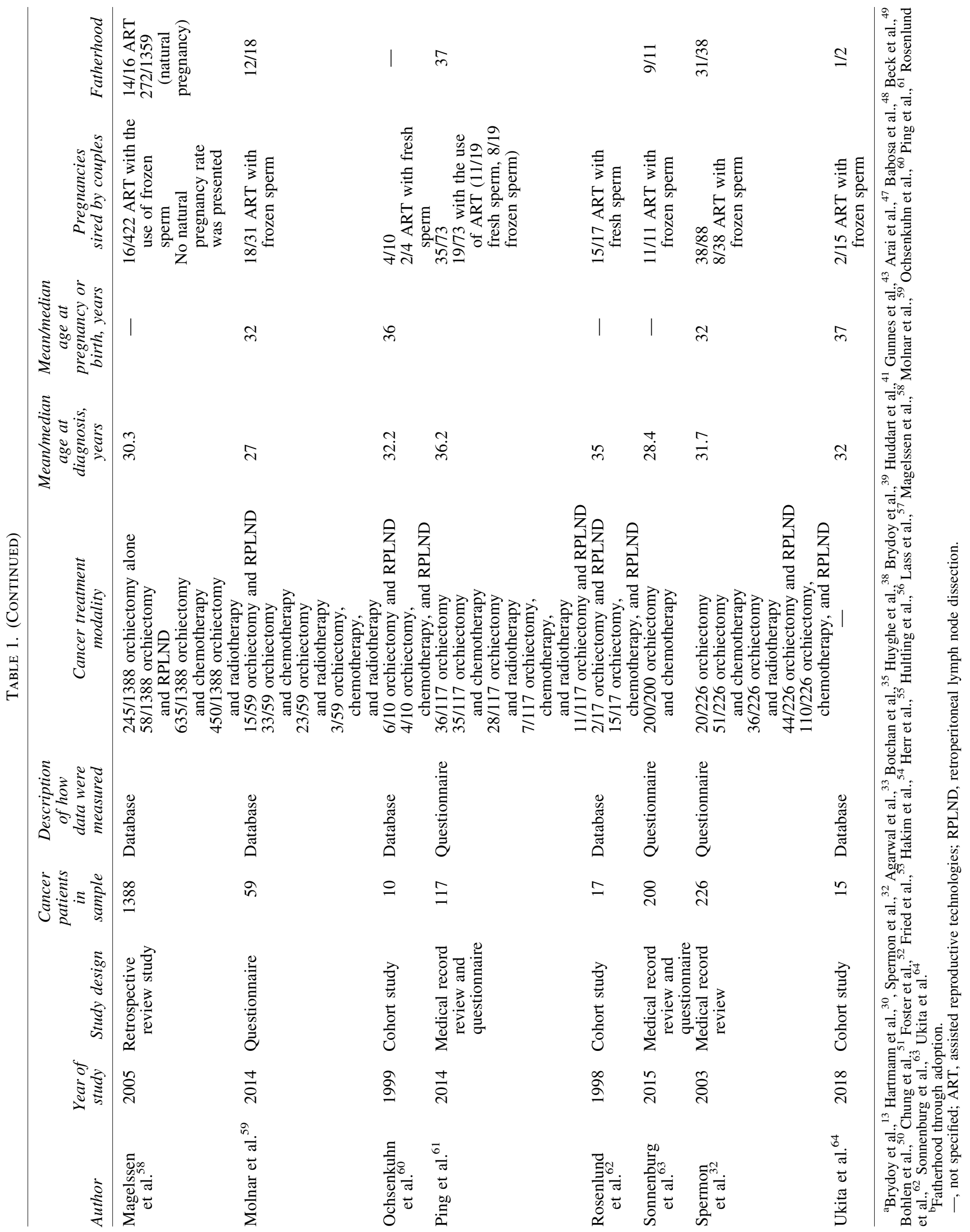


Of those articles that reported on TGCT type and stage for male's diagnosed with a seminoma $(n=16$ articles), ${ }^{13,30,32,33,35,38,39,41,43,47-64} 24.37 \%$ were stage $1^{41,53}$ followed by $8.83 \%$ who presented with stage $2 .{ }^{41,53}$ No staging was reported for the other $14(66.8 \%)$ studies for this TGCT type.

For those articles that reported on nonseminomas ( $n=19$ articles), $13,30,32,38,41,43,47-50,54,55,57-63$ there were 3861 men diagnosed with this TGCT, including: teratomas $(n=26 / 312,8.33 \%)^{38,48,54,60,62}$ embryonal $(n=190 / 478$, $39.75 \%),{ }^{38,48,50,54,61,62}$ choriocancers $(n=7 / 274,2.55 \%),{ }^{38,48}$ yolk sac $(n=8 / 380,2.11 \%), 38,54,61,62$ and mixed tumors $(n=152 / 362,41.99 \%) .^{38,60,62}$ Only six studies reported on stage of TC; stage $1(369 / 1330,27.74 \%),{ }^{30,41,49,50,55}$ stage 2 $(64 / 285,22.46 \%),{ }^{30,49,54}$ stage $3(17 / 98,17.35 \%),{ }^{30}$ and stage unknown $(32.45 \%)$.

\section{Reproductive outcomes, in relation to fatherhood, following treatment for TC}

Table 2 highlights reproductive outcomes, in relation to fatherhood, following treatment for a testicular malignancy for the combined studies. ${ }^{13,30,32,33,35,38,39,41,43,47-64}$

The rate of azoospermia, before starting treatment, reported in 11 articles, $32,50,52,55-59,61,62,64$ was $7.24 \%(n=138 /$

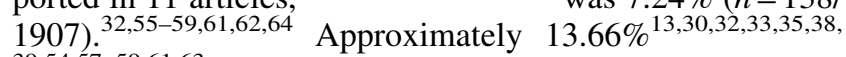
$39,54,57-59,61,63$ of male patient's banked sperm and $52.29 \%$ $(n=2834 / 5420)^{13,30,32,39,47,55,58,59,61-63}$ of couples attempted to conceive subsequent to treatment.

Subgroup analysis. There were 6439 males included in the combined articles, with a total of $1192(22 \%)(95 \% \mathrm{CI}$ : $\left.0.21-0.23 ; \quad I^{2}=98.1 \%\right)^{13,30,32,35,38,39,41,43,47,51,52,54,56-62,64}$ conceptions following treatment for TC (Fig. 2) (median age at diagnosis was 30.7 years and at pregnancy or birth was 34 years). Table 2 details the number of conceptions, in relation to different cancer treatment options, following cancer therapy. There were $31.8 \%(n=214)^{13,30,32,33,35,38,39,49,51,54,56-64}$ of couples that became pregnant using ART. There was only 1 article that reported on adoption in $27(7.24 \%, n=27 / 373)^{13}$ males.

Pooled miscarriage $(<14$ weeks) and medical terminations for couples were $11 \%(n=25)\left(95 \% \mathrm{CI}: 0.07-0.16 ; I^{2}=\right.$ $8.5 \%)^{30,32,48,52,56,60-62,64}$ and $10 \%(n=21)(95 \%$ CI: $0.05-$ $\left.0.15 ; I^{2}=57 \%\right),{ }^{48,52,58,59,61}$ respectively.

Of those couples that successfully conceived, fatherhood (live birth) was experienced by $37 \%(n=993)$ (95\% CI: $0.35-$ $\left.0.39 ; I^{2}=98.1 \%\right)^{13,30,32,33,35,39,48-50,52,53-55,58,59,63}$ of males following TC (Fig. 3).

There were no data reported on preterm births $(<37$ weeks), still births, or perinatal deaths included in any of the studies, hence only pregnancy, terminations, miscarriages, and live birth data have been reported.

Treatment with an orchiectomy alone. There were eight studies that reported on fatherhood following treatment with an orchiectomy alone (Table 2). There were $684(20 \%)$ (95\% CI: $\left.0.19-0.21 ; I^{2}=98.7 \%\right)^{13,30,32,41,47,57,58,61}$ males who received this treatment who successfully conceived with their partner. Of those males that experienced a pregnancy with their partner, 753 (32\%) (95\% CI: 0.31-0.34; $\left.I^{2}=99.1 \%\right)^{13,30,32,33,55,58}$ had a live birth. The median age at

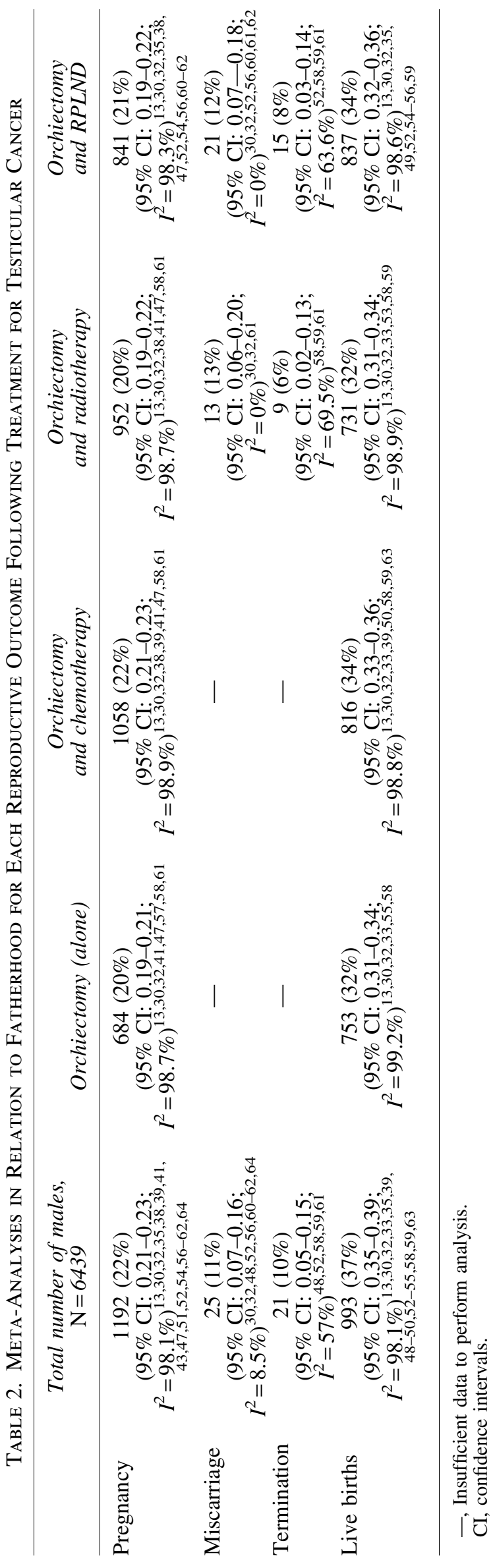


Author

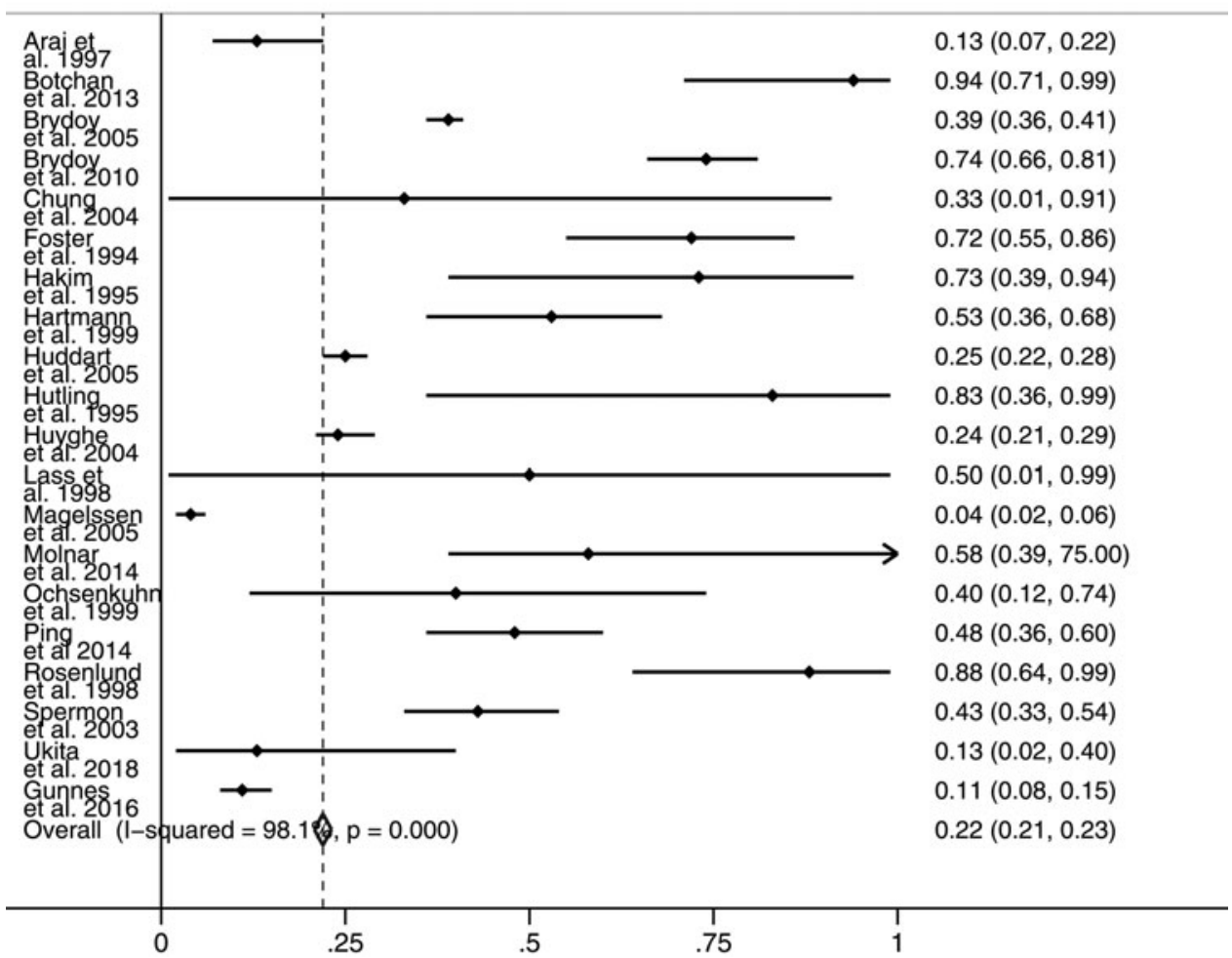

FIG. 2. Number of pregnancies sired with a partner following treatment for TC $(n=1319) . \quad$ TC, testicular cancer. cancer diagnosis and at birth was 30.3 and 35 years, respectively, for these collective studies.

Treatment with an orchiectomy followed by chemotherapy. There were 1058 (22\%) (95\% CI: $\left.0.21-0.23 ; I^{2}=98.9 \%\right)^{13,30 \text {, }}$
$32,38,39,41,47,58,61$ males that received combined treatment with an orchiectomy followed by chemotherapy that experienced a pregnancy with their partner. Of those couples that successfully conceived, $34 \%(n=816)$ (95\% CI: $0.33-0.36$; $\left.I^{2}=98.8 \%\right)^{13,30,32,33,39,50,58,59,63}$ resulted in a live birth
Author

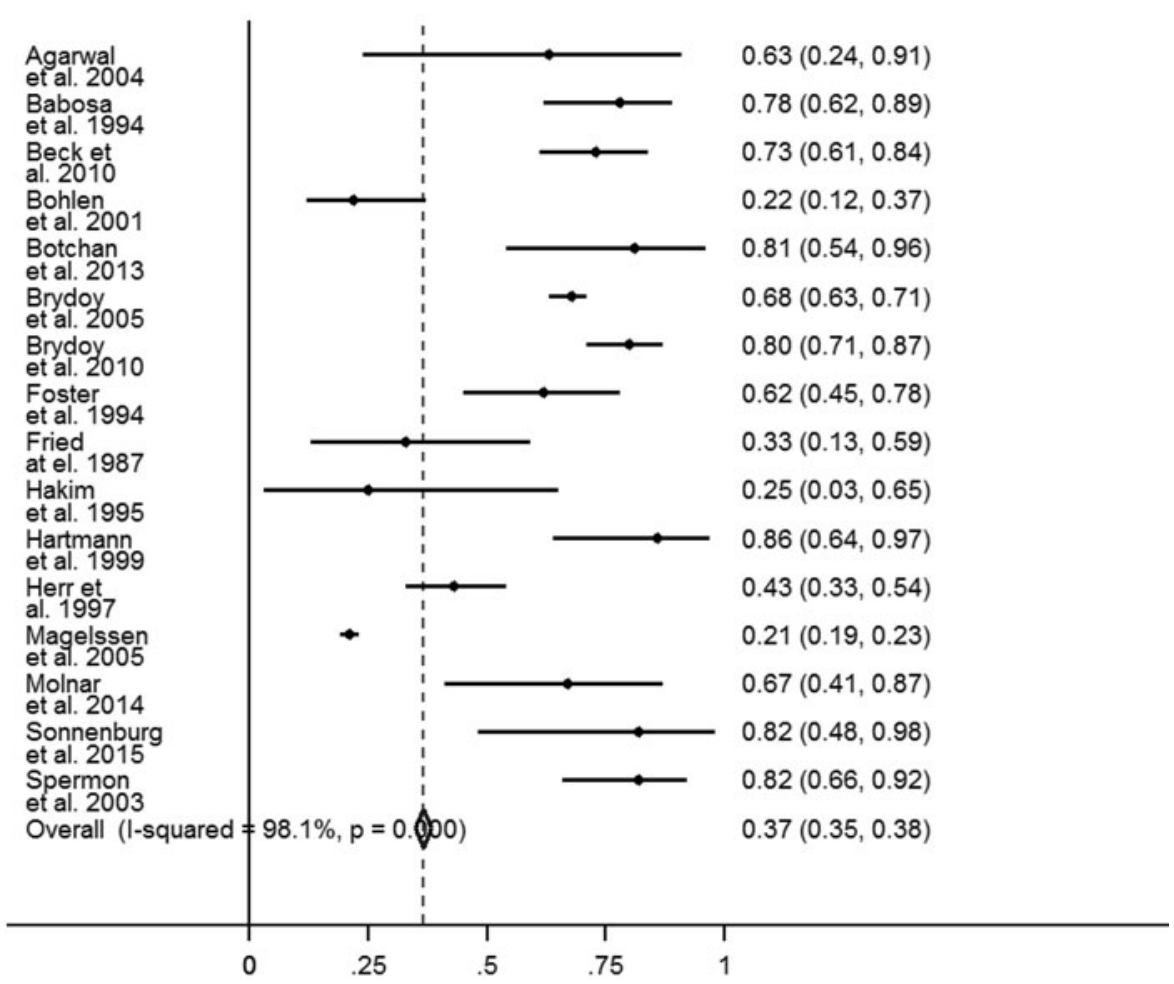

FIG. 3. Fatherhood following treatment for TC $(n=993)$. 
(median age at cancer diagnosis and at pregnancy or birth were 28.7 and 30.3 years, respectively).

Treatment with an orchiectomy followed by radiotherapy. For those males who had received multimodality treatment with an orchiectomy followed by radiotherapy, similarly low pregnancy rates of around 20\% $(n=952)(95 \%$ CI: 0.19-0.22; $\left.I^{2}=98.7 \%\right)^{13,30,32,38,41,47,58,61}$ were experienced by couples with $32 \%(n=731)\left(95 \% \text { CI: } 0.31-0.34 ; I^{2}=98.9 \%\right)^{13,30 \text {, }}$ $32,33,53,58,59$ experiencing a live birth. Median age at diagnosis and at pregnancy or birth was 29.7 and 32 years, respectively.

Treatment with an orchiectomy followed by RPLND. Additionally, males who received combination treatment with an orchiectomy followed by a RPLND, were $21 \%$

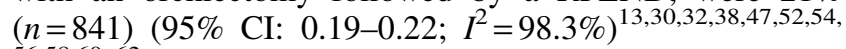
$56,58,60-62$ likely to achieve pregnancy with their partner and $34 \%(n=837)\left(95 \% \text { CI: } 0.32-0.36 ; I^{2}=98.6 \%\right)^{13,30,32,49,52 \text {, }}$ $54,-56,58,59$ of couples experienced parenthood subsequent to treatment. Median age at diagnosis and at pregnancy or birth was 31.9 and 34.5 years, respectively.

\section{Discussion}

This systematic review and meta-analysis provide evidence that low pregnancy and live birth rates for couples are associated with treatment for TC. ${ }^{32}$ Additionally, some male cancer patients may still desire fatherhood, but may not be ready to start a family at the time of treatment or alternatively may have already completed having a family. As TC is considered the most common cancer affecting males of reproductive age and with increasing curative rates and survival for TC, patients need to be given an opportunity to consider their fertility in relation to their diagnosis and treatment.

Current clinical guidelines ${ }^{65-70}$ recommend that newly diagnosed male cancer patients be referred for counseling and discussions regarding infertility as part of comprehensive cancer care in relation to their diagnosis and treatment, including those who may have already fathered children. Hence, the importance of having discussions and counseling around sperm banking before the start of treatment. ${ }^{63}$ Several studies have also indicated elevated fertility-related psychological distress in cancer survivors when their fertility is impacted following treatment, ${ }^{71}$ hence the importance of timely referrals for fertility discussions.

Approximately $14 \%^{13,30,32,33,35,38,39,54,57-59,61,63}$ of male patients in this study underwent sperm banking and these rates are in line with other studies reporting similarly low rates for sperm banking of cancer patients between $10 \%$ and $20 \%{ }^{58,63}$ Barriers associated with poor uptake for sperm banking may include lack of referral to a reproductive specialist, poor specialist knowledge regarding effects of TC treatment on fertility, cost of sperm banking, and patient/clinician wanting to start treatment urgently. Data presented in this article should therefore be interpreted with caution as not all patients may have desired fatherhood for many personal reasons or may have unsuccessfully attempted to conceive with their partner and these data may not have been captured. Additionally, we did not have data on the number of males who were not offered sperm banking or those who were referred for fertility preservation and decided not to cryopreserve their sperm.

Meta-analyses were performed using pooled data from the combined studies. Finding of this meta-analysis indicated an overall conception rate of $22 \%(n=1192)$ for couples who conceived after TC, ${ }^{13,30,32,35,38,39,41,43,47,51,52,54,56-62,64}$ although more than half of all couples (52.29\%) attempted parenthood following treatment. ${ }^{13,30,32,39,47,55,58,59,61-63}$ Use of ART for conception was reported in $31.8 \%$ couples (with either intracytoplasmic sperm injection-in vitro fertilization [ICSI-IVF] or intrauterine insemination [IUI]). $13,30,32,33,35,38,39,49,51,54,56-64$

The opportunity for achieving fatherhood has significantly improved over the last 5 years with advancements in fertility preservation techniques and increased awareness of both semen and testicular sperm extraction for cryopreservation. These techniques in combination with ART of IVF or ICSI ${ }^{33,72,73}$ have helped to improve fertility outcomes for couples.

However, it should be noted that cancer patients may present with infertility concerns unrelated to their cancer diagnosis (such as low sperm production, abnormal sperm function, impotence, diabetes, erection and ejaculation difficulties and blockages that prevent the delivery of sperm, and injuries) before starting treatment. These men are at greatest risk for infertility.

In addition, we performed subanalysis to explore reproductive rates for fatherhood in relation to different TC treatment modalities. It was interesting to observe that most couples had $<25 \%$ (19-23\%) chance of siring a pregnancy with their partner following TC treatment with an orchiectomy alone and/or with chemotherapy, radiotherapy, or a RPLND. The literature reports that infertility has been estimated to occur in about $25 \%$ of TC survivors within the first $2-5$ years posttreatment. $^{74,75}$ It has also been reported that reproductive cancer treatments often result in significant fertility reductions, most noticeable in the first few years following treatment.

However, as insufficient information was reported on chemotherapeutic agents as well as dose and site for administration of radiotherapy, in the included studies, we were unable to present fertility outcomes in relation to specific treatment regimens.

It has been well reported in the literature that chemotherapy regimens and radiotherapy can have deleterious effects on male fertility and endocrine function, particularly on spermatogenesis. ${ }^{76,77}$ The effects of TC treatment on sperm DNA and chromatin during the recovery process is important as damage to the paternal genome can have devastating effects on the offspring. ${ }^{76}$ There are debatable outcomes reported in the literature associated with alterations in sperm DNA. With one study highlighting an increase in DNA damage 6-24 months subsequent to treatment, ${ }^{78}$ conversely another study reported no increase in DNA damage. ${ }^{79}$

A study by Bujan et al. ${ }^{76}$ reported adverse gonadotoxic effects, particularly with the use of chemotherapy and radiotherapy, on spermatogenesis of TC patients, with potential recovery at 2 years post-treatment. The study reported elevated effects of radiotherapy on chromatin changes at 6 months after cancer treatment with an increase in aneuploidy during the 17 months following the use of chemotherapeutic agents, such as bleomycin, etoposide, and cisplatin (BEP), which are used to treat cancer patients. ${ }^{80}$ These outcomes have been confirmed in results, highlighted in other studies, following the first 2 years post-treatment. ${ }^{81-85}$ 
Ghezzi et al. ${ }^{77}$ assessed the impact of having an orchiectomy followed by chemotherapy (BEP treatment, or surveillance alone) on sperm parameters, sperm aneuploidies and DNA fragmentation, sex hormones, and testicular volume in TGCT cancer patients. Patients $(n=212)$ were followed at various time points (baseline 12- and 24-months post-treatment) to determine the effects of carboplatin (CARB) compared with surveillance and BEP treatments. Findings indicated an increase of sperm aneuploidies after BEP therapy up until 24 months post-treatment and adverse effects on sperm DNA, which continued up until 24 months from end of treatment. The authors also highlighted that one cycle of CARB did not have an effect on sperm aneuploidies, DNA compaction, and DNA fragmentation.

Overall, the estimated medical termination rate was $10 \% .^{48,52,58,59,61}$ Couples may decide to terminate a pregnancy following cancer treatment as they may experience heightened psychological concerns and fears associated with their diagnosis. Additionally, spermatozoa may carry damaged DNA after completion of treatment, hence the concern that cancer survivors may transmit a defective genome to their offspring. ${ }^{84}$ Furthermore, cancer patients may also fear disease relapse and how this may affect their ability to parent. ${ }^{86}$

Furthermore, we conducted a subanalysis on fatherhood (live birth rates). Less than half of all couples who became pregnant after TC treatment experienced a live birth $(37 \%)$ (95\% CI: $\left.0.35-0.39 ; I^{2}=97.8 \%\right)^{13,30,32,33,35,39,48-56,58,59,63,64}$ Unfortunately, as only five studies ${ }^{48,52,58,59,61}$ reported data on medical terminations, we were unable to determine the actual proportion of live births that may have been experienced.

The literature reports that infertility affects $\sim 20-30 \%$ of males and $15 \%$ of couples globally. ${ }^{87}$ Rates for fatherhood reported in this study are considerably lower compared with males from the general population. Fossa and Kravdal ${ }^{88}$ report that males treated for TC experience lower fertility rates of around $30 \%$ compared with those males in the general population.

Tang et al. ${ }^{89}$ report that male cancer survivors were $23 \%$ (standard birth ratios: $0.77,95 \% \mathrm{CI}$ : 0.75-0.79) less likely to father an offspring compared with males without cancer in the general population. ${ }^{89}$ Furthermore, these authors report that males diagnosed with a childhood cancer $(0-12$ years) are $38 \%$ less likely to give birth (standardized birth ratios [SBRs] 0.62, 95\% CI: 0.57-0.67) compared with males diagnosed with cancer in adolescence or adulthood. They also highlight that males diagnosed with cancer in adulthood are $20 \%$ less likely to experience parenthood (SBR 0.80, 95\% CI: $0.78-0.82$ ) compared with the general population of males without cancer.

Additionally, Syse et al. ${ }^{90}$ report similar findings, where male cancer survivors had a $24 \%$ (odds ratio $0.76,95 \%$ CI: 0.72-0.79) lower first birth experience compared with the general population when matched by age and education. Similar findings were presented by Green et al. ${ }^{91}$ who stated that male survivors were $44 \%$ (hazard ratio $0.56,95 \% \mathrm{CI}$ : 0.49-0.63) less likely to conceive with their partner compared with siblings who had not undergone cancer treatment.

\section{Limitations}

There are several limitations that need to be addressed in presenting reproductive outcomes, in relation to fatherhood, from the combined studies. Data were unavailable in some of the studies in relation to cancer stage, baseline semen analysis (number of sperm, motility, morphology, volume of the ejaculated sample), number of patients that relapsed following initial treatment for TC and only few studies provided time to pregnancy or birth following TC treatment. Also rates for partner infertility were not included in the studies. Additionally, as control groups were not included in each of the studies, we were unable to conduct odds or risk ratios for aged-matched groups (cancer vs. control) in relation to reproductive outcomes for each study. Despite these limitations associated with this study, we still report several interesting findings.

\section{Conclusions}

This systematic review and meta-analysis report low pregnancy and birth rates for male TC survivors who desire fatherhood as compared with those males in the general population. We present findings in support of international guidelines that recommend patients be referred for discussions around treatment-related fertility impairment before starting cancer treatment ${ }^{33}$ as well as oncofertility psychosocial support.

\section{Author Contributions}

B.G. and A.A. were responsible for the conception and design of this study. B.G., A.K., M.B., and X.V. were responsible for screening included studies. B.G., O.A., and H.W. were responsible for the analysis of data. All authors read and approved the final article.

\section{Availability of Data and Materials}

All data pertaining to this study are contained and presented in this article.

\section{Author Disclosure Statement}

No competing financial interests exist.

\section{Funding Information}

No funding was received for this article.

\section{References}

1. Huyghe E, Matsuda T, Thonneau P Increasing incidence of testicular cancer worldwide: a review. J Urol. 2003;170(1): 5-11.

2. Coleman M, Gatta G, Verdecchia A, et al. EUROCARE-3 summary: cancer survival in Europe at the end of the 20th century. Ann Oncol. 2003;14(Suppl 5):v128-49.

3. La Vignera S, Cannarella R, Duca Y, Barbagallo F, et al. Hypogonadism and sexual dysfunction in testicular tumor survivors: a systematic review. Front Endocrinol (Lausanne). 2019;10:264.

4. McGlynn KA, Cook MB. Etiologic factors in testicular germ-cell tumors. Future Oncol. 2009;5(9):1389-402.

5. Curado M-P, Edwards B, Shin HR, et al. Cancer incidence in five continents, Vol IX. Lyon, France: IARC Press, International Agency for Research on Cancer; 2007.

6. Carrière $P$, Baade $P$, Fritschi L. Population based incidence and age distribution of spermatocytic seminoma. J Urol. 2007;178(1):125-8. 
7. Tournaye H, Dohle GR, Barratt CL. Fertility preservation in men with cancer. Lancet. 2014;384(9950):1295-301.

8. Lampe H, Horwich A, Norman A, et al. Fertility after chemotherapy for testicular germ cell cancers. J Clin Oncol. 1997;15(1):239-45.

9. Fosså SD, Åbyholm T, Normann N, et al. Post-treatment fertility in patients with testicular cancer: III. Influence of radiotherapy in seminoma patients. Br J Urol. 1986; 58(2-4):315-9.

10. Berthelsen JG. Sperm counts and serum follicle-stimulating hormone levels before and after radiotherapy and chemotherapy in men with testicular germ cell cancer. Fertil Steril. 1984;41(2):281-6.

11. Pont J, Albrecht W. Fertility after chemotherapy for testicular germ cell cancer Fertil Steril. 1997;68(1):1-5.

12. Nijman J, Koops HS, Oldhoff J, et al. Sexual function after bilateral retroperitoneal lymph node dissection for nonseminomatous testicular cancer. Arch Androl. 1987;18(3): 255-67.

13. Brydoy M, Fossa SD, Klepp O, et al. Paternity following treatment for testicular cancer. J Natl Cancer Inst. 2005; 97(21):1580-8.

14. Donohue J, Thornhill J, Foster R, et al. Retroperitoneal lymphadenectomy for clinical stage A testis cancer (1965 to 1989): modifications of technique and impact on ejaculation. J Urol. 1993;149(2):237-43.

15. Peckham M, Husband J, Barrett A, et al. Orchidectomy alone in testicular stage I non-seminomatous germ-cell tumours. Lancet. 1982;320(8300):678-80.

16. Fosså SD, Horwich A, Russell J, et al. Optimal planning target volume for stage I testicular seminoma: a Medical Research Council randomized trial. J Clin Oncol. 1999; 17(4):1146-6.

17. Jones WG, Fossa SD, Mead GM, et al. Randomized trial of 30 versus $20 \mathrm{~Gy}$ in the adjuvant treatment of stage I testicular seminoma: a report on Medical Research Council Trial TE18, European Organisation for the Research and Treatment of Cancer Trial 30942 (ISRCTN18525328). J Clin Oncol. 2005;23(6):1200-8.

18. De Wit R, Roberts JT, Wilkinson PM, et al. Equivalence of three or four cycles of bleomycin, etoposide, and cisplatin chemotherapy and of a 3-or 5-day schedule in goodprognosis germ cell cancer: a randomized study of the European Organization for Research and Treatment of Cancer Genitourinary Tract Cancer Cooperative Group and the Medical Research Council. J Clin Oncol. 2001;19(6): 1629-40.

19. Presti JC, Herr HW, Carroll PR. Fertility and testis cancer. Urol Clin North Am. 1993;20(1):173-9.

20. Turek PJ, Lowther DN, Carroll PR. Fertility issues and their management in men with testis cancer. Urol Clin North Am. 1998;25(3):517-31.

21. Meirow D, Schenker J. Infertility: cancer and male infertility. Hum Reprod. 1995;10(8):2017-22.

22. Stephenson WT, Poirier SM, Rubin L, et al. Evaluation of reproductive capacity in germ cell tumor patients following treatment with cisplatin, etoposide, and bleomycin. J Clin Oncol. 1995;13(9):2278-80.

23. Matos E, Škrbinc B, Zakotnik B. Fertility in patients treated for testicular cancer. J Cancer Surviv. 2010;4(3):274-8.

24. Benson R, Beard C, Kelalis P, et al. Malignant potential of the cryptorchid testis. In: Mayo Foundation for Medical Education and Research. Mayo Clinic Proceedings. New York: Elsevier; 1991; pp. 372-8.
25. Lee PA. Fertility in cryptorchidism: does treatment make a difference? Endocrinol Metab Clin North Am. 1993;22(3): 479-90.

26. Brennemann W, Stoffel-Wagner B, Helmers A, et al. Gonadal function of patients treated with cisplatin based chemotherapy for germ cell cancer. J Urol. 1997;158(3): 844-50.

27. Yoon J, Park H, Ju H, et al. Gonadal and sexual dysfunction in childhood cancer survivors. Cancer Res Treat. 2017; 49(4):1057-64.

28. Anazodo A, Ataman-Millhouse L, Jayasinghe Y, et al. Oncofertility - an emerging discipline rather than a special consideration. Pediatr Blood Cancer. 2018;65(11):e27297.

29. Kenney L, Antal Z, Ginsberg J, et al. Improving male reproductive health after childhood, adolescent, and young adult cancer: progress and future directions for survivorship research. J Clin Oncol. 2018;36(21):2160-8.

30. Hartmann JT, Albrecht C, Schmoll HJ, et al. Long-term effects on sexual function and fertility after treatment of testicular cancer. Br J Cancer. 1999;80(5-6):801-7.

31. Logan S, Perz J, Ussher JM, et al. A systematic review of patient oncofertility support needs in reproductive cancer patients aged 14 to 45 years of age. Psychooncology. 2018; 27(2):401-9.

32. Spermon JR, Kiemeney LA, Meuleman EJ, et al. Fertility in men with testicular germ cell tumors. Fertil Steril. 2003; 79(Suppl 3):1543-9.

33. Agarwal A, Ranganathan P, Kattal N, et al. Fertility after cancer: a prospective review of assisted reproductive outcome with banked semen specimens. Fertil Steril. 2004; 81(2):342-8.

34. Hallak J, Kolettis PN, Sekhon VS, et al. Sperm cryopreservation in patients with testicular cancer. Urology. 1999; 54(5):894-9.

35. Botchan A, Karpol S, Lehavi O, et al. Preservation of sperm of cancer patients: extent of use and pregnancy outcome in a tertiary infertility center. Asian J Androl. 2013;15(3):382-6.

36. Fosså SD, Aass N, Molne K. Is routine pre-treatment cryopreservation of semen worthwhile in the management of patients with testicular cancer? Br J Urol. 1989;64(5): 524-9.

37. Van Casteren N, van Santbrink E, Van Inzen W, et al. Use rate and assisted reproduction technologies outcome of cryopreserved semen from 629 cancer patients. Fertil Steril. 2008;90(6):2245-50.

38. Huyghe E, Matsuda T, Daudin M, et al. Fertility after testicular cancer treatments: results of a large multicenter study. Cancer. 2004;100(4):732-7.

39. Brydoy M, Fossa SD, Klepp O, et al. Paternity and testicular function among testicular cancer survivors treated with two to four cycles of cisplatin-based chemotherapy. Eur Urol. 2010;58(1):134-40.

40. Kim C, McGlynn KA, McCorkle R, et al. Fertility among testicular cancer survivors: a case-control study in the US. J Cancer Surviv. 2010;4(3):266-73.

41. Huddart RA, Norman A, Moynihan C, et al. Fertility, gonadal and sexual function in survivors of testicular cancer. Br J Cancer. 2005;93(2):200-7.

42. Honecker F, Aparicio J, Berney D, et al. ESMO Consensus Conference on testicular germ cell cancer: diagnosis, treatment and follow-up. Ann Oncol. 2018;29(8):1658-86.

43. Gunnes MW, Lie RT, Bjørge T, et al. Reproduction and marriage among male survivors of cancer in childhood, 
adolescence and young adulthood: a national cohort study. Br J Cancer. 2016;114(3):348.

44. DerSimonian R, Laird N. Meta-analysis in clinical trials. Control Clin Trials. 1986;7(3):177-88.

45. Higgins JP, Thompson SG, Deeks JJ, et al. Measuring inconsistency in meta-analyses. BMJ. 2003;327(7414):557-60.

46. Cook MB, Akre O, Forman D, et al. A systematic review and meta-analysis of perinatal variables in relation to the risk of testicular cancer-experiences of the son. Int $\mathrm{J}$ Epidemiol. 2010;39(6):1605-18.

47. Arai Y, Kawakita M, Okada Y, et al. Sexuality and fertility in long-term survivors of testicular cancer. J Clin Oncol. 1997;15(4):1444-8.

48. Babosa M, Baki M, Bodrogi I, et al. A study of children, fathered by men treated for testicular cancer, conceived before, during, and after chemotherapy. Med Pediatr Oncol. 1994;22(1):33-8.

49. Beck SD, Bey AL, Bihrle R, et al. Ejaculatory status and fertility rates after primary retroperitoneal lymph node dissection. J Urol. 2010;184(5):2078-80.

50. Bohlen D, Burkhard FC, Mills R, et al. Fertility and sexual function following orchiectomy and 2 cycles of chemotherapy for stage I high risk nonseminomatous germ cell cancer. J Urol. 2001;165(2):441-4.

51. Chung K, Irani J, Knee G, et al. Sperm cryopreservation for male patients with cancer: an epidemiological analysis at the University of Pennsylvania. Eur J Obstet Gynecol Reprod Biol. 2004;113(Suppl 1):S7-11.

52. Foster RS, McNulty A, Rubin LR, et al. The fertility of patients with clinical stage I testis cancer managed by nerve sparing retroperitoneal lymph node dissection. J Urol. 1994;152(4):1139-42.

53. Fried P, Steinfeld R, Casileth B, et al. Incidence of developmental handicaps among the offspring of men treated for testicular seminoma. Int J Androl. 1987;10(1):385-7.

54. Hakim LS, Lobel SM, Oates RD. The achievement of pregnancies using assisted reproductive technologies for male factor infertility after retroperitoneal lymph node dissection for testicular carcinoma. Fertil Steril. 1995; 64(6):1141-6.

55. Herr HW, Bar-Chama N, O'Sullivan M, et al. Paternity in men with stage I testis tumors on surveillance. J Clin Oncol. 1998;16(2):733-4.

56. Hultling C, Rosenlund B, Tornblom M, et al. Transrectal electroejaculation in combination with in-vitro fertilization: an effective treatment of anejaculatory infertility after testicular cancer. Hum Reprod. 1995;10(4):847-50.

57. Lass A, Akagbosu F, Abusheikha N, et al. A programme of semen cryopreservation for patients with malignant disease in a tertiary infertility centre: lessons from 8 years' experience. Hum Reprod. 1998;13(11):3256-61.

58. Magelssen H, Haugen TB, von During V, et al. Twenty years experience with semen cryopreservation in testicular cancer patients: who needs it? Eur Urol. 2005;48(5):779-85.

59. Molnar Z, Berta E, Benyo M, et al. Fertility of testicular cancer patients after anticancer treatment-experience of 11 years. Pharmazie. 2014;69(6):437-41.

60. Ochsenkuhn R, Kamischke A, Nieschlag E. Imipramine for successful treatment of retrograde ejaculation caused by retroperitoneal surgery. Int J Androl. 1999;22(3):173-7.

61. Ping P, Gu BH, Li P, Huang YR, et al. Fertility outcome of patients with testicular tumor: before and after treatment. Asian J Androl. 2014;16(1):107-11.
62. Rosenlund B, Sjöblom P, Törnblom M, et al. In-vitro fertilization and intracytoplasmic sperm injection in the treatment of infertility after testicular cancer. Hum Reprod. 1998;13(2):414-8.

63. Sonnenburg DW, Brames MJ, Case-Eads S, et al. Utilization of sperm banking and barriers to its use in testicular cancer patients. Support Care Cancer. 2015;23(9): 2763-8.

64. Ukita Y, Wakimoto Y, Sugiyama Y, et al. Fertility preservation and pregnancy outcomes in adolescent and young adult male patients with cancer. Reprod Med Biol. 2018; 17(4):449-53.

65. American Society of Clinical Oncology. ASCO recommendations on fertility preservation in cancer patients: guideline summary. J Oncol Pract. 2006;2:143.

66. Loren AW, Mangu PB, Beck LN, et al. Fertility preservation for patients with cancer: American Society of Clinical Oncology clinical practice guideline update. J Clin Oncol. 2013;31(19):2500-10.

67. Schrader M, Muller M, Sofikitis N, et al. "Onco-tese": testicular sperm extraction in azoospermic cancer patients before chemotherapy-new guidelines? Urology. 2003; 61(2):421-5.

68. Health Improvement Scotland Long term follow up of survivors of childhood cancer. A national clinical guideline. 2013. Accessed July 10, 2019 from: www.sign.ac.uk/ pdf/sign132.pdf

69. National Institute for Health Care and Excellence Fertility: assessment and treatment for people with fertility problems. Accessed July 10, 2019 from: http://publications.nice.org .uk/fertility-cg156

70. Cancer Council Australia [homepage on the Internet] Sydney: Cancer Council Australia; Accessed July 10, 2019 from: www.cancer.org.au

71. Logan S, Perz J, Ussher JM, et al. Systematic review of fertility-related psychological distress in cancer patients: informing on an improved model of care. Psychooncology. 2019;28(1):22-30.

72. Wallace WHB, Anderson RA, Irvine DS. Fertility preservation for young patients with cancer: who is at risk and what can be offered? Lancet Oncol. 2005;6(4): 209-18.

73. Dohle GR. Male infertility in cancer patients: review of the literature. Int J Urol. 2010;17(4):327-31.

74. Howell SJ, Shalet SM. Spermatogenesis after cancer treatment: damage and recovery. J Natl Cancer Inst Monogr. 2005;2005(34):12-7.

75. Chaudhary UB, Haldas JR. Long-term complications of chemotherapy for germ cell tumours. Drugs. 2003;63(15): $1565-77$.

76. Bujan L, Walschaerts $\mathrm{M}$, Moinard $\mathrm{N}$, et al. Impact of chemotherapy and radiotherapy for testicular germ cell tumors on spermatogenesis and sperm DNA: a multicenter prospective study from the CECOS network. Fertil Steril. 2013;100(3):673-80.

77. Ghezzi M, Berretta M, Bottacin A, et al. Impact of BEP or carboplatin chemotherapy on testicular function and sperm nucleus of subjects with testicular germ cell tumor. Front Pharmacol. 2016;7:122.

78. O'Flaherty C, Hales BF, Chan P, et al. Impact of chemotherapeutics and advanced testicular cancer or Hodgkin lymphoma on sperm deoxyribonucleic acid integrity. Fertil Steril. 2010;94(4):1374-9. 
79. Smit M, van Casteren NJ, Wildhagen MF, et al. Sperm DNA integrity in cancer patients before and after cytotoxic treatment. Hum Reprod. 2010;25(8):1877-83.

80. De Mas P, Daudin M, Vincent M-C, et al. Increased aneuploidy in spermatozoa from testicular tumour patients after chemotherapy with cisplatin, etoposide and bleomycin. Hum Reprod. 2001;16(6):1204-8.

81. Tempest HG, Ko E, Chan P, et al. Sperm aneuploidy frequencies analysed before and after chemotherapy in testicular cancer and Hodgkin's lymphoma patients. Hum Reprod. 2008;23(2):251-8.

82. Martin RH, Ernst S, Rademaker A, et al. Analysis of sperm chromosome complements before, during, and after chemotherapy. Cancer Genet Cytogenet. 1999;108(2): 133-6.

83. Zhou BB, Bartek J. Targeting the checkpoint kinases: chemosensitization versus chemoprotection. Nat Rev Cancer. 2004;4(3):216-25.

84. Li Z, Yang J, Huang H. Oxidative stress induces H2AX phosphorylation in human spermatozoa. FEBS Lett. 2006; 580(26):6161-8.

85. Paoli D, Gallo M, Rizzo F, et al. Testicular cancer and sperm DNA damage: short- and long-term effects of antineoplastic treatment. Andrology. 2015;3(1):122-8.

86. Winther JF, Boice Jr JD, Svendsen AL, et al. Induced abortions in Danish cancer survivors: a population-based cohort study. J Natl Cancer Inst. 2009;101(9):687-9.
87. Agarwal A, Mulgund A, Hamada A, et al. A unique view on male infertility around the globe. Reprod Biol Endocrinol. 2015;13(1):37.

88. Fossa SD, Kravdal O. Fertility in Norwegian testicular cancer patients. Br J Cancer. 2000;82(3):737-41.

89. Tang S-W, Liu J, Juay L, et al. Birth rates among male cancer survivors and mortality rates among their offspring: a population-based study from Sweden. BMC Cancer. 2016;16(1):196.

90. Syse A, Kravdal $\varnothing$, Tretli S. Parenthood after cancer-a population-based study. Psychooncology. 2007;16(10): 920-7.

91. Green DM, Kawashima T, Stovall M, et al. Fertility of male survivors of childhood cancer: a report from the Childhood Cancer Survivor Study. J Clin Oncol. 2010; 28(2):332-9.

Address correspondence to: Antoinette Anazodo, MBBS, PhD

Kids Cancer Centre

Sydney Children's Hospital

Randwick

Sydney, NSW 2031

Australia

E-mail: antoinette.anazodo@ health.nsw.gov.au 